
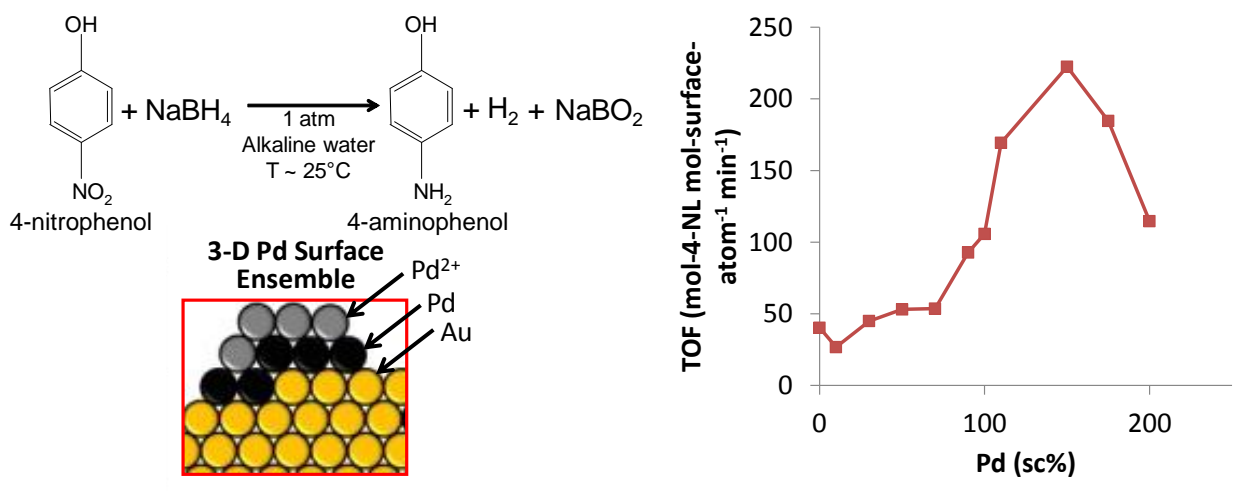


\title{
Improving Gold Catalysis of Nitroarene Reduction with Surface Pd
}

\author{
Lori A. Pretzer, ${ }^{1}$ Kimberly N. Heck, ${ }^{2}$ Sean S. Kim, ${ }^{2}$ Yu-Lun Fang, ${ }^{2}$ Zhun Zhao, ${ }^{2}$ Neng Guo, \\ Tianpin Wu, ${ }^{4}$ Jeffrey T. Miller, ${ }^{4}$ and Michael S. Wong ${ }^{1,2,3,5^{*}}$ \\ ${ }^{1}$ Department of Chemistry, ${ }^{2}$ Department of Chemical and Biomolecular Engineering, \\ ${ }^{3}$ Department of Civil and Environmental Engineering, Rice University, Houston, TX 77005 \\ ${ }^{4}$ Chemical Sciences and Engineering Division, Argonne National Laboratory, Lemont, IL 60439 \\ ${ }^{5}$ Department of Material Science and Nanoengineering, Rice University, Houston, TX, 77005
}

Nitroarene reduction reactions are commercialized catalytic processes that play a key role in the synthesis of many products including medicines, rubbers, dyes, and herbicides. Whereas bimetallic compositions have been studied, a better understanding of the bimetallic structure effects may lead to improved industrial catalysts. In this work, the influence of surface palladium atoms supported on 3-nm $\mathrm{Au}$ nanoparticles (Pd-on-Au NPs) on catalytic activity for 4nitrophenol reduction is explored. Batch reactor studies indicate Pd-on-Au NPs exhibit maximum catalytic activity at a Pd surface coverage of $150 \mathrm{sc} \%$, with an initial turnover frequency of $\sim 3.7$ mol-nitrophenol $/$ mol-metal surface $_{1} / \mathrm{s}$, which was $\sim 5.5 \times$ and $\sim 13 \times$ more active than pure Au NPs and Pd NPs, respectively. Pd NPs, Au NPs, and Pd-on-Au NPs below 175 sc\% show compensation behavior. Three-dimensional Pd surface ensembles (with 4-5 atoms) previously identified through $\mathrm{x}$-ray adsorption spectroscopy provide the active sites responsible for the catalytic maximum. These results demonstrate the ability to adjust systematically a structural feature (i.e., Pd surface coverage) to yield a more active material.

*Author to whom correspondence should be addressed. Email: mswong@ rice.edu, Telephone: (713) 348-3511, Fax: (713) 348-5478

Keywords: nitrophenol, catalysis, palladium, gold, nanoparticle, bimetallic, structure 


\subsection{Introduction.}

Pd-on-Au nanoparticles (Pd-on-Au NPs) are a model catalyst consisting of Au NPs with controllable amounts of Pd atoms deposited on the NP surface[1,2]. The amount of deposited Pd, calculated as the Pd surface coverage ("sc", dictates the size of the Pd surface ensembles and extent of Pd oxidation [3, 4]. They have been studied extensively for a number of aqueous-phase reactions, i.e., the hydrodechlorination (HDC) of trichloroethene [1-5] and perchloroethene [6]), the reduction of nitrite anions [7], and, most recently, the partial oxidation of glycerol [8, 9]. For all these reactions, catalytic activity followed a volcano-shape dependence on Pd surface coverage, with maximum activities occurring when Pd surface coverage was less than $100 \%$ (equivalent to 1 calculated Pd monolayer). Enhanced activity for chloroform HDC was also observed when $\mathrm{Pd}$ atoms were deposited on $\mathrm{Au} / \mathrm{Al}_{2} \mathrm{O}_{3}$ catalysts [10].

This work seeks to strengthen the understanding of Pd-on-Au catalysis by studying the NPs for 4-nitrophenol ("4-NL") reduction. This model reaction generates 4-aminophenol ("4AP") selectively [11-13] in the presence of a metal catalyst using sodium borohydride as a reductant (scheme 1).[11, 14, 15] It can be carried out at room temperature and atmospheric pressure, and it is usually monitored through ultraviolet-visible spectroscopy, making it an ideal model reaction and the subject of review articles by Ballauff and coworkers[16] and Pal and coworkers.[17]

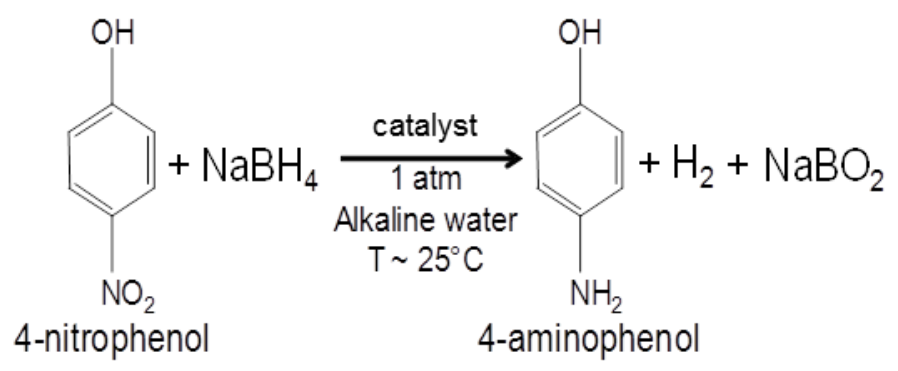

Scheme 1. Chemical equation for the catalytic reduction of 4-nitrophenol (4-NL) using sodium borohydride. 
There is industrial interest in aromatic amines like 4-NL because they have widespread industrial uses for medicines, dyes and stains, pesticides, and explosives [18-20]. They are commercially produced through the Bechamp process (which involves using an acidic slurry of zerovalent iron reagent at $\sim 75-100{ }^{\circ} \mathrm{C}[21]$ ) and metal catalysis (which involves $\mathrm{Pt}$, Pd, or Rh, and hydrogen at $\sim 100-110{ }^{\circ} \mathrm{C}$ and $\sim 20-60$ atm [22]).

Several studies on PdAu composition effects on 4-NL reduction have been reported. Kaiser et al. synthesized a series of PdAu alloy NPs by co-reducing Pd and Au precursor salts. The activity of all the bimetallic catalysts was found to be higher than monometallic Au or Pd NPs, and was maximum at a Au mole fraction of $75 \mathrm{~mol} \%$ (exhibiting $10 \times$ higher activity than the monometallic NPs).[15] These results qualitatively agree with a study by Tang et al., who studied PdAu alloy NPs formed via sequential reduction of Au precursor salt onto a Pd catalyst. This group found that increasing mole fraction Au also led to increased activity, with a molar ratio of $\mathrm{Au}: \mathrm{Pd}$ of 2.1:1 (or equivalent $\mathrm{Au}$ mole fraction of $67 \mathrm{~mol} \%$ ) being most active. The maximum activity was $\sim 3 \times$ higher compared to their monometallic Pd catalyst [23]. A study by Zhao et al. on a series of PdAu catalysts synthesized via coreduction showed the same higher activity of the bimetallic catalysts, though maximum activity occurred for the composition of $\mathrm{Au}: \mathrm{Pd}$ molar ratio of 3:7 (30 mol\% $\mathrm{Au}$ ) [24]. While bulk metal composition can be controlled, the differences in catalysis indicate differences in the bimetal structure.

Pd-on-Au NPs with well-characterized Pd surface structures and a single Au particle size can offer additional insights into 4-NL reduction. In this work, batch reaction studies of a series of colloidal $\sim 3 \mathrm{~nm}$ NPs with different Pd surface coverages were carried for 4-NL reduction with sodium borohydride. Reaction rate constants and initial turnover frequencies were determined at room temperature (from 0 to $200 \mathrm{sc} \%$, surface coverage), and compared with the Pd surface 
structures previously determined from x-ray adsorption spectroscopy (XAS). New XAS analysis was carried out on Pd-on-Au NPs with a higher Pd surface coverage than previously studied (i.e., $200 \mathrm{sc} \%$ ). The activation energy and preexponential factor values were determined in the temperature range of $30-60{ }^{\circ} \mathrm{C}$, and discussed in the context of a compensation effect.

\subsection{Materials and Methods.}

\subsection{Materials}

Palladium(II) chloride $\left(\mathrm{PdCl}_{2} ;>99.99 \%\right)$, gold (III) chloride trihydrate $\left(\mathrm{HAuCl}_{4} \cdot 3 \mathrm{H}_{2} \mathrm{O}, 99 \%\right)$, tannic acid (>99.5\%), potassium carbonate (>99.5\%), and 4-nitrophenol (4-NL, >99\%), were purchased from Sigma-Aldrich. Sodium citrate dihydrate $(>99.5 \%)$ was obtained from Fischer Scientific. Sodium borohydride (>98\%) was purchased from Acros (Lot number: B0116503). Sodium hydroxide (1.0M) was obtained from EMD Chemicals. Amorphous carbon powder (Vulcan XC-72 and Monarch 700) was purchased from Cabot. Ultra-high purity argon gas, nitrogen gas, and hydrogen gas were obtained from Matheson Tri-gas. Deionized (DI) water from a Barnstead NANOpure Diamond purifier (resistivity $>18 \mathrm{M} \Omega / \mathrm{cm}$ ) was used for all experiments. All chemicals were used as received unless otherwise noted.

\subsection{Au NP synthesis}

3-nm $\mathrm{Au}$ NPs were synthesized as previously reported.[4, 25] A $0.32 \mathrm{mM}$ chloroauric acid $\left(\mathrm{HAuCl}_{4}\right)$ solution was added to a reductant solution $(1.5 \mathrm{mM}$ tannic acid, $7.0 \mathrm{mM}$ trisodium citrate dihydrate, and $6.3 \mathrm{mM}$ potassium carbonate), with both pre-heated to a temperature of 60 ${ }^{\circ} \mathrm{C}$ prior to mixing. The resulting brown sol was vigorously stirred and heated to a boil. After boiling for two min, the sol was cooled to room temperature and the total sol volume was 
adjusted to $100 \mathrm{~mL}$ with additional DI water. The NP size was verified using transmission electron microscopy.[4] Au NPs were stored at $4{ }^{\circ} \mathrm{C}$ for no longer than 1 week before use.

\subsection{Pd-on-Au NP synthesis}

Pd-on-Au NPs with Pd surface coverages between 10 and 200\% were synthesized by reducing aliquots (13-265 $\mu \mathrm{L})$ of a $2.47 \mathrm{mM}$ chloropalladic (II) acid $\left(\mathrm{H}_{2} \mathrm{PdCl}_{4}\right)$ solution with hydrogen gas onto the Au NP sols under ambient conditions as previously described (table S1).[4] The volume of $2.47 \mathrm{mM} \mathrm{H}_{2} \mathrm{PdCl}_{4}$ needed for a specific Pd surface coverage was calculated using the magic cluster model, in which each Au NP is viewed as a central Au atom surrounded by consecutive shells of Au atoms.[2, 26, 27] A 3-nm Au NP would consist of 5 consecutive Au shells, 561 total $\mathrm{Au}$ atoms, and 252 surface Au atoms (table S2). A surface coverage of 100\% is then modeled as a $6^{\text {th }}$ consecutive shell on the NP since Pd and Au atoms are nearly the same size. Surface coverages exceeding 100\% refer to additional Pd layers, e.g., "200 sc\%" means that a 3-nm Pdon-Au NP is modeled as a magic cluster of 5 Au shells and 2 Pd shells.[4] The concentration of NPs in the $3 \mathrm{~nm}$ Au NP sol was calculated to be $2.7 \times 10^{14} \mathrm{NPs} / \mathrm{mL}$. Elemental analysis indicated complete $\mathrm{HAuCl}_{4}$ reduction and showed good agreement between calculated and actual Pd metal content.[4] The Au NP size has previously been found to be $3.0 \pm 0.7 \mathrm{~nm}$, and TEM confirmed no significant change in particle size (i.e., beyond the initial Au particle size distribution) or size distribution when Pd was deposited on the Au NP surface.[4]

\subsection{Pd NP Synthesis}

The activity of Pd-on-Au NPs was compared to those of pure $3 \mathrm{~nm}$ and 4-nm Pd NPs. The synthesis of pure Pd NPs $(\sim 4 \mathrm{~nm})$ has been reported in detail.[2] Briefly, Pd NPs were synthesized using an experimental protocol similar to the Au NPs except that a $0.32 \mathrm{mM} \mathrm{PdCl}_{4}{ }^{2-}$ 
solution (12 mL of $2.39 \mathrm{mM} \mathrm{PdCl}_{4}{ }^{2-}$ diluted with $68 \mathrm{~mL} \mathrm{H}_{2} \mathrm{O}$ ) was used. Pd sols were heated to boiling for $\sim 25$ min prior to cooling to room temperature under ambient conditions.[4] 


\section{$2.4 X$-ray absorption spectroscopy}

X-ray absorption spectroscopy of carbon-supported 200\% Pd NPs was conducted at the Advanced Photon Source at Argonne National Laboratory. Details concerning the XAS experiment protocol can be found in previous work.[3, 4]

\subsection{Catalytic testing}

A $10 \mathrm{mM}$ 4-nitrophenol stock solution was made by adding $41.7 \mathrm{mg}$ of 4-NL to $30 \mathrm{~mL}$ of water and sonicating for $20 \mathrm{~min}$. An alkaline $0.1 \mathrm{M} \mathrm{NaBH}_{4}$ stock solution was made by dissolving $0.038 \mathrm{~g}$ of $\mathrm{NaBH}_{4}$ in $10 \mathrm{~mL}$ of an ice-cold $0.1 \mathrm{mM} \mathrm{NaOH}$ solution $(100 \mu \mathrm{L}$ of $0.01 \mathrm{M} \mathrm{NaOH}$ diluted with $9.9 \mathrm{~mL}$ water). A fresh $\mathrm{NaBH}_{4}$ stock solution was made every 3 h. Catalytic testing was conducted in a closed batch reactor, in which a screw-cap quartz cuvette (1-cm path length) is filled with $2.7 \mathrm{~mL}$ water, $30 \mu \mathrm{L}$ of $10 \mathrm{mM}$ 4-NL stock solution, and $200 \mu \mathrm{L}$ of $0.1 \mathrm{M} \mathrm{NaBH}_{4}$ stock solution and sealed with a PTFE-silicone septum. The sealed cuvette was purged with an inert gas (either $\operatorname{Ar}$ or $\mathrm{N}_{2}$ ) for 1 min to displace dissolved oxygen and fill the headspace ( 1 atm).

An aliquot $(51 \mu \mathrm{L})$ of as-prepared 3-nm Pd-on-Au NP sols was diluted with $9.94 \mathrm{~mL}$ of water to prepare a diluted NP sol (total Au M concentration $=1.3 \times 10^{-6} \mathrm{M} ; 260 \mathrm{ppb}$ ). The total Pd concentration ranged from $=8.4 \times 10^{-8} \mathrm{M}(8.9 \mathrm{ppb})$ to $1.7 \times 10^{-6} \mathrm{M}(180 \mathrm{ppb})$, depending on the $\mathrm{Pd}$ surface coverage. The diluted NP sol $(100 \mu \mathrm{L})$ was then injected into the quartz cuvette at $\mathrm{t}=0$ min after the cuvette was purged with inert gas. The final concentration of Pd-on-Au NPs in the reactor was $4.5 \times 10^{10} \mathrm{NP} / \mathrm{mL}$. No UV-vis absorbance from the NPs was observed at this concentration. The final concentration of total $\mathrm{Au}$ in the reactor was $4.2 \times 10^{-8} \mathrm{M}$ while the total final Pd concentration ranged from $2.7 \times 10^{-9} \mathrm{M}$ to $5.5 \times 10^{-8} \mathrm{M}$.

Monitoring of the 4-NL reduction reaction was started $30 \mathrm{~s}$ after NP injection using UVvis spectroscopy, in which the absorbance of the 4-NL nitro group at $\lambda=400 \mathrm{~nm}$ (red-shifted from 
$\sim 313 \mathrm{~nm}$ due to basic reaction conditions) was measured.[28] Initial concentrations of 4-NL and $\mathrm{NaBH}_{4}$ were $9.7 \times 10^{-2} \mathrm{mM}(\sim 13.5 \mathrm{ppm})$ and $6.5 \mathrm{mM}(\sim 246 \mathrm{ppm})$, respectively. These initial reactant concentrations were within range of other published studies.[11, 12, 14, 15] All reactions were monitored for total $\mathrm{t}=26.5 \mathrm{~min}$ using a Shimadzu 2450 UV-Vis spectrophotometer. Reaction spectra were acquired every 2 min. Between UV-vis scans, the quartz cuvette was mixed using a Fisher Scientific mini vortexer set at $\sim 7$. The reaction was determined to not be diffusion-limited under these experimental conditions based on calculated Damköhler number $\left(D_{a}\right)$ values $[16,29] ; D_{a}<0.0015$ for all the NP compositions tested, in good agreement with similar calculations by Ballauff and co-workers (see Supporting Information).

The catalytic reduction reaction can be modeled as a Langmuir-Hinshelwood mechanism in which adsorbed 4-NL and borohydride species react to form 4-AP, metaborate $\left(\mathrm{BO}_{2}{ }^{-}\right)$, and hydrogen.[12, 15, 16, 30]. Since the $\mathrm{NaBH}_{4}$ concentration greatly exceeds $(\sim 65 \times)$ that of 4-NL, the reaction rate constants were calculated from a pseudo-first order rate law with respect to 4NL $[11,12,14,31,32]$ :

$$
-\mathrm{dC}_{4-\mathrm{NL}} / \mathrm{dt}=\mathrm{k}_{\text {meas }} \mathrm{C}_{4-\mathrm{NL}}
$$

where $\mathrm{C}_{4-\mathrm{NL}}$ is the 4-NL concentration and $\mathrm{k}_{\text {meas }}$ is the measured pseudo-first-order rate constant. Each experiment was carried out at least three times.

Initial turnover frequency (TOF) values were calculated by normalizing the measured rate constant to the total concentrations of surface $\mathrm{Au}$ atoms $\left(\mathrm{C}_{\mathrm{Au} \text { surface atoms }}\right)$ and surface $\mathrm{Pd}$ atoms $\left(\mathrm{C}_{\mathrm{Pd} \text { surface atoms }}\right)$ :

$$
\mathrm{TOF}=\left(\mathrm{k}_{\text {meas }} \mathrm{C}_{4-\mathrm{NL}} /\left[\left(\mathrm{C}_{\mathrm{Pd} \text { surface atoms }}\right)+\left(\mathrm{C}_{\text {Au surface atoms }}\right)\right]\right)
$$

For NPs with $<100 \mathrm{sc} \%$, the $\mathrm{C}_{\mathrm{Pd} \text { surface atoms }}$ comes from the number of Pd atoms in the $6^{\text {th }}$ shell of the magic cluster model and the $\mathrm{C}_{\mathrm{Au}}$ surface atoms comes from the number of exposed $\mathrm{Au}$ atoms in 
the $5^{\text {th }}$ shell. For NPs with $>100 \mathrm{sc} \%, \mathrm{C}_{\mathrm{Pd} \text { surface atoms }}$ comes from the number of Pd atoms in the $7^{\text {th }}$ shell and the exposed Pd atoms in the $6^{\text {th }}$ shell. The concentration of Pd and Au surface atoms used for reported TOFs is provided in Table S3. The concentration of Pd and Au metal used in all batch reactor experiments were such that the observed reaction rates varied linearly with catalyst charge, as shown in figures S1-S4. The $\mathrm{pH}$ of the reaction solution $(\sim 10.4)$ did not change during the reaction. No reaction was observed in the absence of catalyst NPs.

Control experiments with monometallic Pd and Au NPs were performed as follows. Approximately $100 \mu \mathrm{L}$ of a diluted $\sim \mathrm{nm}$ Pd NP sol (446 $\mu \mathrm{L}$ as prepared Pd NP sol diluted with $9.54 \mathrm{~mL} \mathrm{H}_{2} \mathrm{O}$ ) was injected into the sealed quartz reactor (final Pd concentration in reactor =

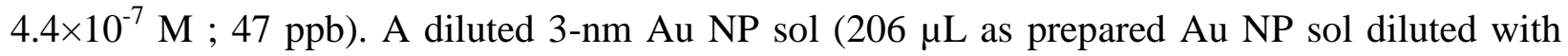
$9.78 \mathrm{~mL} \mathrm{H}_{2} \mathrm{O}$ ) was prepared and $100 \mu \mathrm{L}$ of the diluted sol was injected into the quartz reactor (final Au concentration in reactor $=1.7 \times 10^{-7} \mathrm{M} ; 34 \mathrm{ppb}$ ). UV-vis absorbance by the NPs at these concentrations was negligible.

Activation energy values were determined for $3 \mathrm{~nm}$ Au NPs, $4 \mathrm{~nm}$ Pd NPs, and selected Pd-on-Au NPs by determining $\mathrm{k}_{\text {meas }}$ in the temperature range of $30-60{ }^{\circ} \mathrm{C}$ at $10{ }^{\circ} \mathrm{C}$ increments for each catalyst. The reactor temperature was controlled using a water-jacketed sample chamber located in the UV-Vis spectrometer, with the maximum temperature variation in the batch reactor observed to be $\pm 1{ }^{\circ} \mathrm{C}$ during a typical catalytic experiment. Thermal equilibration between the batch reactor and sample chamber occurred within $\sim 30 \mathrm{sec}$, and no significant change in batch reactor temperature $\left(<1{ }^{\circ} \mathrm{C}\right)$ was observed due to vortexing between UV-vis measurements. The activation energy and frequency factor of each catalyst was determined using the Arrhenius equation. 


\subsection{Results and Discussion.}

\subsection{Nanostructure of Pd-on-Au NPs}

In an earlier report, the metal structure of Pd-on-Au NPs of different calculated Pd surface coverages up to $150 \%$ and at Au NP sizes of 3,7 , and $10 \mathrm{~nm}$ was studied via XAS analysis reported in our previous work.[4] The Pd metal of the 3-nm Pd-on-Au NPs was mostly isolated $\mathrm{Pd}$ atoms at surface coverages $\leq 30 \%$. The Pd was in the form of $2 \mathrm{D}$ ensembles at $\sim 50 \mathrm{sc} \%$, and in the form of 3D ensembles at $>50 \mathrm{sc} \%$. These 3D ensembles also included oxidized Pd, which was inferred to be the Pd atoms located on top of other Pd atoms. With surface coverages increasing from $70 \%$ to $150 \%$, the Pd-Pd coordination numbers increased and the percentage of Pd in the oxidized state increased, suggestive of larger, more aggregated 3D Pd ensembles.[4]

Analogous XAS analysis was extended to 200 sc\% Pd-on-Au NPs (Figure 1). The structure of the Pd-on-Au NPs is based on the XAS-measured Pd and Au coordination numbers (CNs), which are presented in a Punnett square. The details (CNs, bond distances, threshold absorption energies, and Debye-Waller factors, DWF's) for $3 \mathrm{~nm}$ Pd-on-Au NPs with 200\% Pd sc are provided in tables S6-S7. The NPs with 200 sc\% Pd did not have any XAS-detectible $\mathrm{Pd}^{2+}$

a) $\underline{\text { XAS Measured CNs }}$

Scattering atom

\begin{tabular}{cc|c|c|}
\multicolumn{1}{c}{} & \multicolumn{1}{c}{ Au } & Pd \\
\cline { 3 - 4 } $\begin{array}{c}\text { Absorbing } \\
\text { atom }\end{array}$ & Au & 9.1 & 2.1 \\
\cline { 3 - 4 } & Pd & 7.8 & 3.6 \\
\cline { 3 - 4 } & &
\end{tabular}

b) Calculated Alloy CNs

Scattering atom

\begin{tabular}{cc|c|c|}
\multicolumn{1}{c}{} & \multicolumn{1}{c}{ Au } & \multicolumn{1}{c}{ Pd } \\
\cline { 3 - 4 } Absorbing & Au & 4.9 & 6.3 \\
\cline { 3 - 4 } atom & Pd & 5.0 & 6.4 \\
\cline { 3 - 4 } & &
\end{tabular}

Figure 1. (a) XAS-measured CN's for $3 \mathrm{~nm}$ Pd-on-Au NPs with $200 \mathrm{sc} \%$ and (b) calculated CN's for theoretically alloyed Pd-Au NPs (see Eqn. S4-S7) with the same metal content $(56.3 \mathrm{~mol} \%$ $\mathrm{Pd})$, total $\mathrm{Au} \mathrm{CN}$ (Au-Au CN + Au-Pd CN = 11.2), and total Pd CN (Pd-Pd CN + Pd-Au CN = 11.4). Experimental CN's are those of as-prepared nanoparticles. 
The Pd-Au, Pd-Pd, Au-Pd, and Au-Au CN (first atom is the absorber, second atom is the scatterer) of theoretical alloy particles were calculated based on the total Pd and total $\mathrm{Au} \mathrm{CN}$ and the mol\% of $\mathrm{Pd}$ and mol\% of $\mathrm{Au}$ in the particle (see equations S4-S7).[4] A relative comparison of the measured and calculated alloy coordination numbers provides insight into the average structure of the NPs.[3, 4] The measured Au-Au CN ( 9.1) was higher than the calculated alloy $\mathrm{Au}-\mathrm{Au} \mathrm{CN}(\sim 4.9)$ and the measured $\mathrm{Au}-\mathrm{Pd} \mathrm{CN}$ was lower $(\sim 2.1)$ than the calculated alloy $\mathrm{Au}-$ Pd CN ( 6.3), consistent with the NPs being Au-rich in the core and, by inference, Pd-rich at the surface. The measured Pd-Pd CN ( 3.6) was lower than the calculated alloy Pd-Pd CN ( 6.4) and the measured $\mathrm{Pd}-\mathrm{Au} \mathrm{CN}$ was $\sim 7.8$ which was higher than the calculated alloy $\mathrm{Pd}-\mathrm{Au} \mathrm{CN}$ $(\sim 5.0)$.

The lack of $\mathrm{Pd}^{2+}$ is indicative that no $3 \mathrm{D}$ ensembles were present, as oxidized $\mathrm{Pd}$ normally is found with 3D Pd ensembles in Pd-on-Au NPs [4]; indeed, direct contact between Pd and $\mathrm{Au}$ atoms prevents oxidation of surface $\mathrm{Pd}$ due to electronic effects of $\mathrm{Au}$ [33-36]. This implies that, on the surface, the Pd is only present in 2D ensembles (or as isolated atoms). Based on CN similarities, the surface of the $200 \mathrm{sc} \% \mathrm{Pd}-\mathrm{on}-\mathrm{Au}$ NPs resembles that of $30 \mathrm{sc} \% \mathrm{Pd}-\mathrm{on}-\mathrm{Au}$ NPs more than those with a similar loading, such as the $150 \mathrm{sc} \%$ Pd-on-Au NPs which has 3D Pd ensembles [4]. The unexpectedly high Pd-Au CN implies that much of the Pd has likely diffused into the Au NP, which is consistent with the many studies which have observed such Pd interdiffusion into Au surfaces $[33,35,36]$.

\subsection{Activity of Pd-on-Au NPs for 4-nitrophenol reduction}

UV-vis absorbance spectra of the reaction medium were collected every 2 min for 26.5 min for all catalyst compositions, with figure 2 showing the results for 3 compositions: monometallic Au 
NPs, monometallic Pd NPs, and Au NPs with a Pd surface coverage of $150 \%$. No change in 4NL absorbance was observed in the absence of metal NPs.

a)

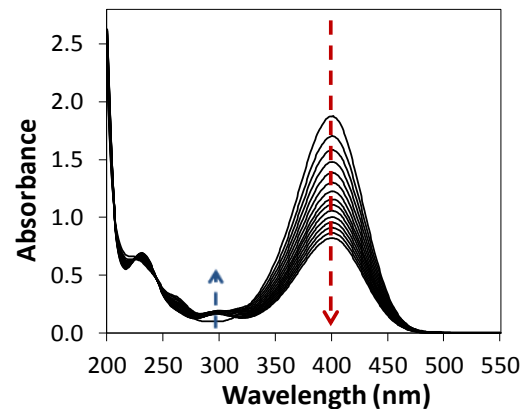

b)

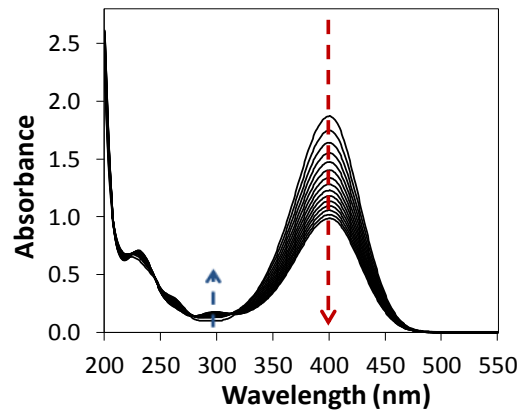

c)

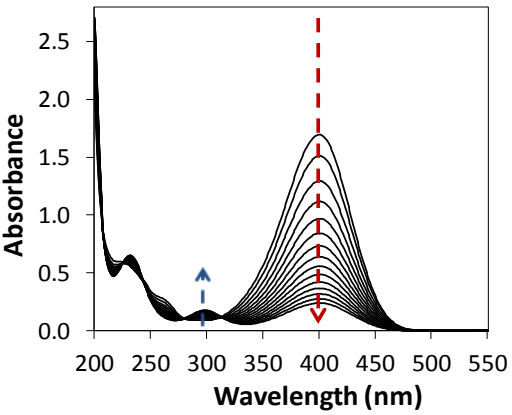

Figure 2. UV-Vis absorbance spectra of a) pure $3 \mathrm{~nm} \mathrm{Au} \mathrm{NPs,} \mathrm{b)} \mathrm{pure} 4 \mathrm{~nm}$ Pd NPs, and c) $3 \mathrm{~nm}$ Pd-on-Au NPs with 150\% Pd sc. The downward (red) and upward (blue) arrows show the decreasing absorbance of 4-NP and increasing absorbance of 4-AP, respectively, as the reaction progresses. Reaction conditions: reaction time $=26.5 \mathrm{~min}$, room temperature $\left(\sim 23{ }^{\circ} \mathrm{C}\right), \mathrm{pH}=$ 10.4, initial 4-NL concentration $=9.7 \times 10^{-2} \mathrm{mM}$, initial $\mathrm{NaBH}_{4}$ concentration $=6.5 \mathrm{mM}$, concentration of a) Au surface atoms $=7.6 \times 10^{-8} \mathrm{M}, \mathrm{b}$ ) Pd surface atoms $=14.8 \times 10^{-8} \mathrm{M}$, and c) total $\mathrm{Pd}$ and $\mathrm{Au}$ surface atoms $=2.9 \times 10^{-8} \mathrm{M}$.

The spectra exhibited isobestic points at $\sim 280 \mathrm{~nm}, \sim 240 \mathrm{~nm}$, and $\sim 220 \mathrm{~nm}$. The presence of these isobestic points in the UV-vis spectra indicated that 4-aminophenol (4-AP) is the only aromatic byproduct formed, as concluded by several studies [11-13, 37]. The principal absorbance wavelengths of 4-NL and 4-AP are $400 \mathrm{~nm}$ and $293 \mathrm{~nm}$, respectively. At the same concentration, 4-AP absorbance is less than 4-NL absorbance because the extinction coefficient of 4-AP is significantly lower than 4-NL at the measured wavelengths. A few research groups observed an induction period attributed to structural rearrangement of the catalyst [12, 29], but this was not observed in our study.

The 4-NL reduction catalytic activity of Au NPs, Pd-on-Au NPs, and Pd NPs is shown in Figure 3. The TOF values of the Au NPs and pure Pd NPs were determined to be 0.67 mol 4-NL mol-Au surface atoms $^{-1} \mathrm{~s}^{-1}\left(=40.3 \mathrm{~mol} 4-\mathrm{NL}\right.$ mol- $\mathrm{Au}_{\text {surface }}$ atoms $\left.^{-1} \mathrm{~min}^{-1}\right)$ and $0.28 \mathrm{~mol} 4-\mathrm{NL}$ mol-Pd $\mathrm{Purface}_{\text {se }}$ atoms $^{-1} \mathrm{~s}^{-1}\left(=16.7 \mathrm{~mol} 4-\mathrm{NL}\right.$ mol-Pd $\left.\mathrm{Purface}_{\text {atoms }}{ }^{-1} \mathrm{~min}^{-1}\right)$, respectively. These values were found to be 
in reasonable agreement with other studies of $\mathrm{Pd}$ and $\mathrm{Au}$ catalyzed 4-NL reduction, after calculating TOF values from published data with the magic cluster model and equation 2 (tables S4 and S5). There was wide range of these literature TOF values (from $\sim 0.2$ to $>200$ mol 4$\mathrm{NL} / \mathrm{mol}_{\text {surface atom }} / \mathrm{min}$ for both $\mathrm{Au}$ and Pd catalysts), due to differences in catalyst support material, particle size, and reaction conditions.

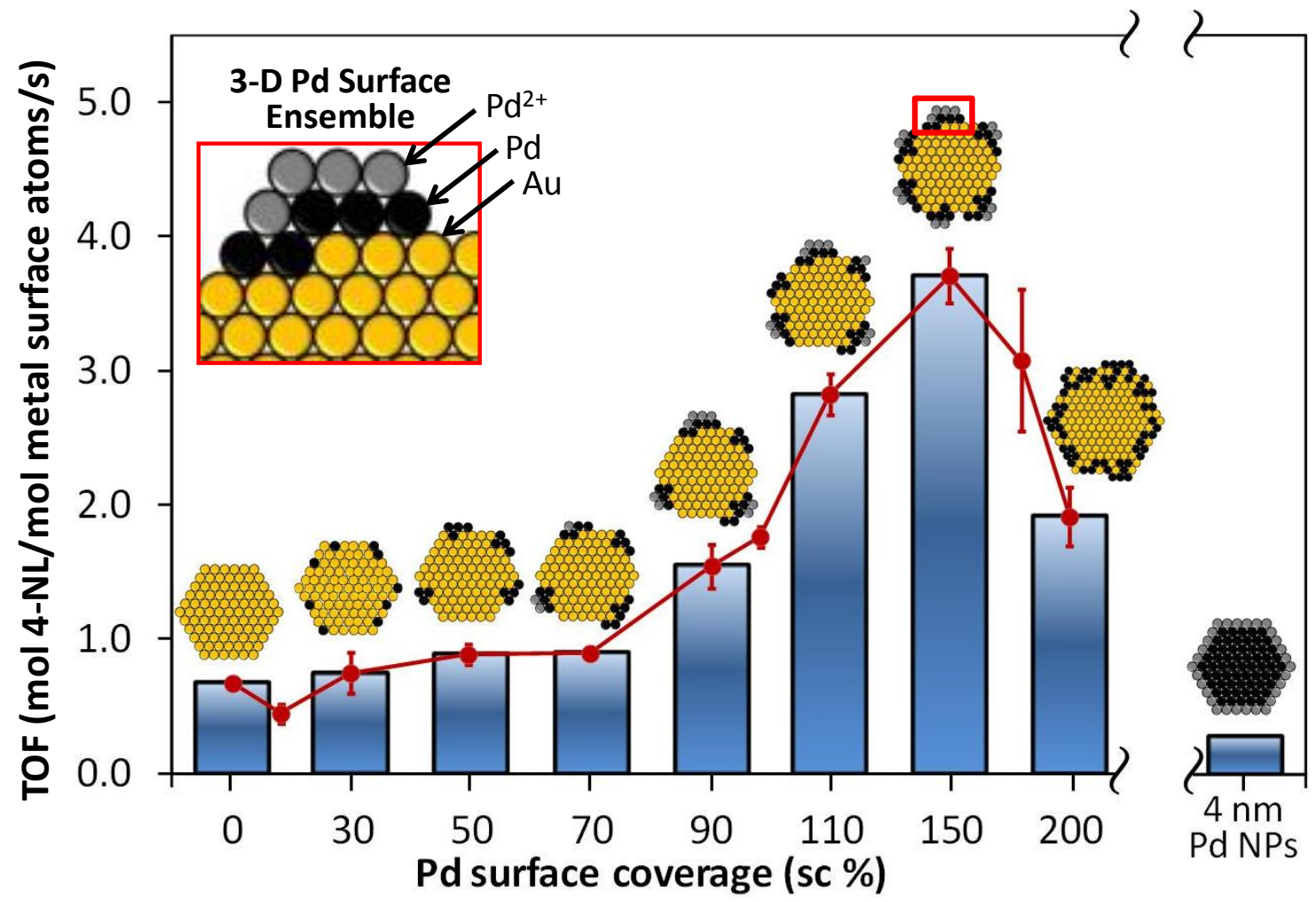

Figure 3. TOF's (shown as bars) and proposed structures of Pd-on-Au NPs with different Pd surface coverages. The complete set of TOF values for Pd surface coverages in the $0-200 \%$ range is shown as the line curve. Cartoons of NP structures representing the XAS results are shown. NPs with $0 \mathrm{sc} \%$ are 3-nm Au NPs. The TOF of 4-nm Pd NPs is included for comparison. Error bars represent the standard deviation of at least three replicate experiments.

Catalytic activity of the Pd-on-Au NPs clearly varied with a volcano-shape dependence on Pd surface coverage. The $30 \mathrm{sc} \%$ sample essentially had the same activity as the pure Au NPs, and the 50 and $70 \mathrm{sc} \%$ samples were $~ 20 \%$ more active. At a Pd surface coverage of $90 \%$ and higher, the TOF values were several times larger than that of Au NPs. The highest activity 
(TOF 3.7 s ${ }^{-1}$ ) was exhibited by Pd-on-Au NPs with $150 \mathrm{sc} \%$, at which Pd forms 3D ensembles. At this surface coverage, these ensembles consist of 4 to $5 \mathrm{Pd}$ atoms on average.[4] At higher surface coverages, the NPs were less active $\left(\sim 3.0 \mathrm{~s}^{-1}\right.$ at $175 \mathrm{sc} \%$ and $\sim 2.0 \mathrm{~s}^{-1}$ at $\left.200 \mathrm{sc} \%\right)$. Through XAFS analysis, at Pd surface coverages greater than $150 \%$ the 3D Pd surface ensembles grow such that extensive Pd-Au surface alloying occurs, with Pd atoms migrating into the Au NP. As a result of such alloying, no Pd oxide is present, and the activity of the NPs with Pd coverages greater than $150 \%$ decreases as geometrically the surface becomes more like the 30-70 sc\% Pd-on-Au NPs, whose surfaces consist primarily of 2D Pd islands.

The enhanced activity of $150 \mathrm{sc} \%$ NPs (with partially oxidized 3D Pd ensembles) is likely due more to the spatial arrangement of the surface Pd atoms (and electronic effect of $\mathrm{Au}$ on Pd affecting adsorption enthalpies of reactants) than the presence of oxidized Pd. The majority (>90\%) of the surface Pd atoms of the pure $4 \mathrm{~nm} \mathrm{Pd} \mathrm{NPs} \mathrm{are} \mathrm{oxidized[3,} \mathrm{4];} \mathrm{hence,} \mathrm{if}$ simply the presence of Pd oxide resulted in significantly higher activity, one would expect the monometallic $4 \mathrm{~nm}$ Pd NPs to exhibit maximum activity. While it is thermodynamically possible for Pd oxide to be reduced by borohydride under our experimental conditions (see equations S1S3),[38] significant particle structure change is unlikely since no induction time is observed.

\subsection{Arrhenius equation analysis.}

The activation energy and pre-exponential factors for catalytic 4-nitrophenol reduction by pure 3 nm Au NPs, pure 4 nm Pd NPs, and 3 nm Pd-on-Au NPs with Pd sc of 70\%, 150\%, 175\%, and $200 \%$ were determined using the logarithmic form of the Arrhenius equation (equation 4):

$$
\ln k_{\text {meas }}=\ln \mathrm{A}-\left(\mathrm{E}_{\mathrm{a}} / \mathrm{RT}\right)
$$

where $\mathrm{A}$ is the pre-exponential factor, $\mathrm{E}_{\mathrm{a}}$ the measured activation energy, $\mathrm{R}$ the gas constant

(8.31 $\left.\mathrm{Jmol}^{-1} \mathrm{~K}^{-1}\right)$, and $\mathrm{T}$ is the temperature $(\mathrm{K})$. The pseudo-first order rate constant $\left(\mathrm{k}_{\text {meas }}\right)$ of 
each of the aforementioned NP samples was measured at $30^{\circ} \mathrm{C}, 40^{\circ} \mathrm{C}, 50^{\circ} \mathrm{C}$, and $60^{\circ} \mathrm{C}$ (see figure S10). Arrhenius plots (ln $k_{\text {meas }}$ vs. $1 / T$ ) were prepared to determine $A$ and $E_{a}$ for each catalyst (see figure S5 and S6).

Figure 4 plots the activation energy results as well as the TOF of the catalysts. As can be seen, the activation energy results for the catalysts are within the range previously reported for metallic catalysts $(7.5 \mathrm{~kJ} / \mathrm{mol}-110 \mathrm{~kJ} / \mathrm{mol})[11,14,39-42]$. The monometallic Au NPs had an activation energy of $12.7 \mathrm{~kJ} / \mathrm{mol}$. A range of activation energies are reported for $\mathrm{Au}$ nanoparticles and are thought to vary with particle shape (38 kJ/mol, Au nanorods) [12], stabilizing agent $(21 \mathrm{~kJ} / \mathrm{mol}$ for citrate stabilized vs. $31 \mathrm{~kJ} / \mathrm{mol}$ for calcium alginate stabilized) [12], and/or support (31 kJ/mol for Au NPs on $\sim 560-\mu \mathrm{m}$ resin beads) [41]. Similarly, the reported activation energies of monometallic Pd NPs range from $7.5 \mathrm{~kJ} / \mathrm{mol}$ (peptide-templated $\sim 3 \mathrm{~nm}$ Pd NPs) [40] to $44 \mathrm{~kJ} / \mathrm{mol}$ ( 2.4 nm Pd NPs in spherical polyelectrolyte brush)[11]. The activation energy of the $4 \mathrm{~nm} \operatorname{Pd} \operatorname{NPs}(\sim 16.3 \mathrm{~kJ} / \mathrm{mol})$ in this work is within this range. The activation energy of $\sim 33-40 \mathrm{~kJ} / \mathrm{mol}$ for the Pd-on-Au NPs (70-200 Pd sc\%) is comparable to activation energies reported for other bimetallic Pd NPs (PtNi NPs=31 kJ/mol, PtPd nanocages $=87-110 \mathrm{~kJ} / \mathrm{mol})[37,43]$. The variation in these activation energies has been ascribed to changes in bimetallic composition and its effect on active site structure. 


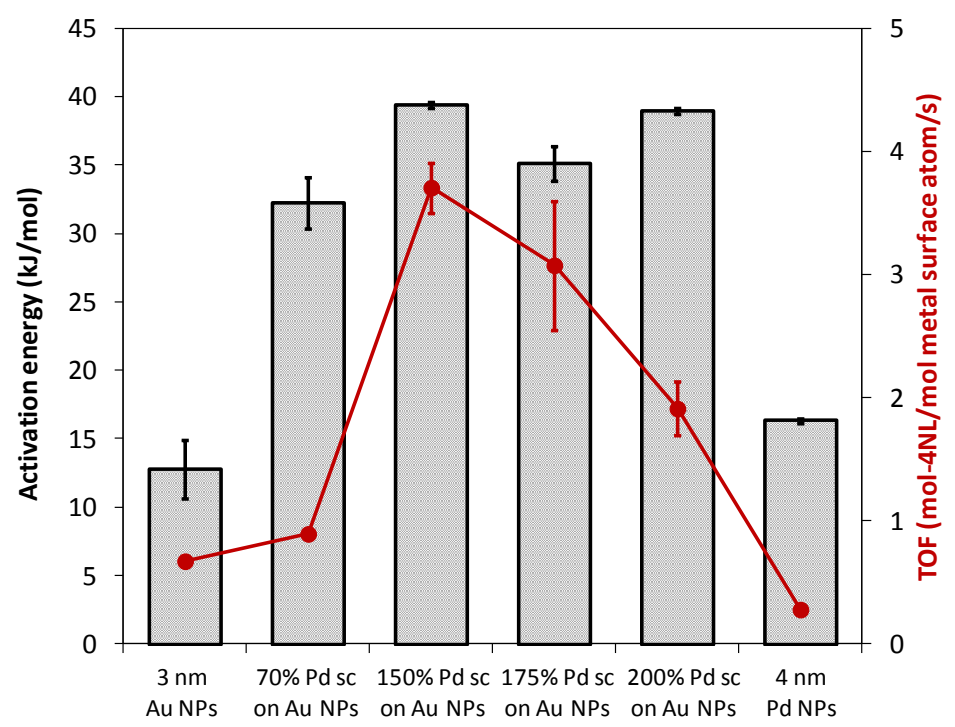

Figure 4. Measured activation energy and TOF of Au NPs, Pd NPs, and selected Pd-on-Au NPs. Error bars represent standard deviations of at least three replicate experiments.

Compensation behavior, in which $\ln \mathrm{A}$ and $\mathrm{E}_{\mathrm{a}}$ follow a linear relationship according to equation 5

$$
\ln \mathrm{A}=\mathrm{aE}_{\mathrm{a}}+\mathrm{b}
$$

where $\mathrm{a}$ and $\mathrm{b}$ are constants [44], has previously been observed for the reduction of 4-NL over metal catalysts $[16,29]$. For the same catalytic mechanism occurring on different catalytic materials, compensation behavior implies that any decrease in the energy barrier for reaction (decrease in $E_{a}$ ) will be counteracted by a decrease in the frequency factor (decrease in $\ln A$ ). That is, the stronger nitrophenol binds to an active site (lower $\ln \mathrm{A}$ ), the more activated it is for reaction (lower $\mathrm{E}_{\mathrm{a}}$ ). [44] Figure 5 shows a Constable plot for the monometallic Pd NPs, monometallic $\mathrm{Au} \mathrm{NPs,}$ and the Pd-on-Au NPs of various surface coverages. As can be seen, there is a linear relationship among the catalysts except for the $200 \mathrm{sc} \% \mathrm{Pd}-\mathrm{on}-\mathrm{Au}$ NPs, which do not show a decrease in $\ln \mathrm{A}$ with $\mathrm{E}_{\mathrm{a}}$. The cause of this deviation is unclear, but could be due to a change in the electronic structure of the active site [45]. This observed compensation behavior arises due to changes in adsorption enthalpies, surface coverage of reactants, and stability of the 
transition state that allow the most active catalyst for this reaction (150 sc\% Pd-on-Au NPs) to have the highest activation energy, which is compensated by its large pre-exponential factor [44]. The Pd-on-Au surface structure leads to higher activation energies and even higher preexponential factors, such that the bimetallic NPs have higher TOF's than the monometallic NPs. The slope of the $\ln \mathrm{A}-\mathrm{E}_{\mathrm{a}}$ curve $\left(\sim 0.40 \ln \left(\mathrm{min}^{-1}\right) \mathrm{mol} \mathrm{kJ}^{-1}\right)$ is similar to that reported by Herves et al. for several different metal catalysts (slope $~ 0.36$ ) [16], indicating that the surface reaction on the NPs in this study (Au, Pd, and Pd-on-Au) follows similar kinetics.[16, 29]

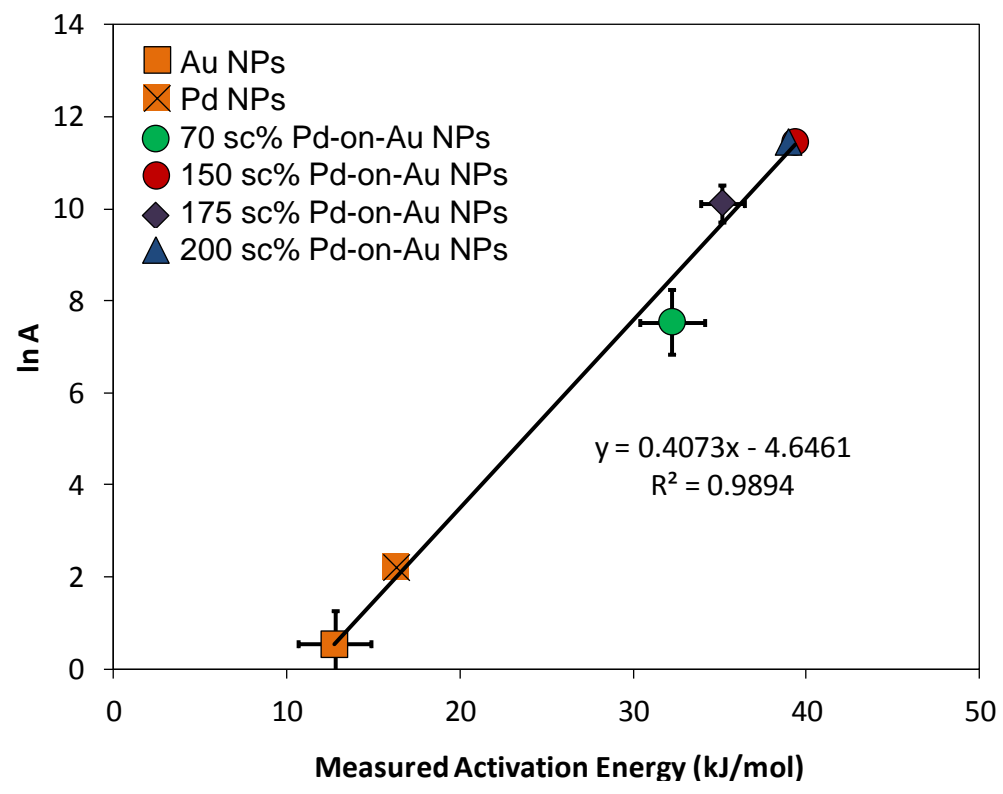

Figure 5. Constable plot of $3 \mathrm{~nm}$ Au NPs, $4 \mathrm{~nm}$ Pd NPs, and representative Pd-on-Au NPs. The particle's activation energy and frequency factors were experimentally determined using the Arrhenius equation. Horizontal and vertical error bars represent the standard deviation of at least three replicate experiments.

\subsection{Conclusions.}

The catalytic reduction of 4-nitrophenol by $\mathrm{NaBH}_{4}$ was studied using a series of model Pd-on-Au NPs with well controlled size and known surface structure. The NP catalysts followed a volcanoshaped dependence on Pd surface coverage and show compensation behavior. Maximum activity on surface atom basis (TOF of $3.7 \mathrm{~s}^{-1}$ ), exhibited by the $150 \mathrm{sc} \%$ Pd-on-Au NP catalyst, 
correlated to the presence of 3D Pd ensembles (consisting of 4-5 Pd atoms) on the $\mathrm{Au} \mathrm{NP}$ surface. It was $\sim 5.5 \times$ and $\sim 13 \times$ higher than that of pure Au NPs $\left(\sim 0.7 \mathrm{~s}^{-1}\right)$ and Pd NPs $\left(\sim 0.3 \mathrm{~s}^{-1}\right)$, respectively. NPs with $200 \mathrm{sc} \%$ were less catalytically active. At this composition, extensive subsurface interdiffusion of Pd was observed, such that the surface sites more closely resemble the less active 2D Pd surface ensembles rather than 3D ensembles. These results show Au NPs can support Pd as surface ensembles up to a certain limit, beyond which Pd cannot be assumed to reside only on the nanoparticle surface.

\subsection{Acknowledgements.}

The authors gratefully acknowledge the National Science Foundation (CBET-1134535) and Welch Foundation (C-1676) for financial support. The authors also thank Dr. J. C. Forsythe, Dr.

Z. L. Schaefer, Dr. N. Soultanidis, Dr. G. C. Kini, Dr. S. Gullapalli, and Mr. J. C. Velazquez for helpful discussions. Use of the Advanced Photon Source was supported by the U. S. Department of Energy, Office of Science, Office of Basic Energy Sciences, under Contract No.DE-AC02$06 \mathrm{CH} 11357$.

\subsection{Supporting Information Available.}

Details behind calculation of mass transfer resistances, detailed synthesis methods of Pd-on-Au NPs (Table S1), magic cluster calculations of Au NPs (Table S2), surface atom concentrations of NPs (Table S3), TOFs of Pd, Au, and Pd-Au catalysts studied by others (Tables S4-S5), details of XAS fitting and results for $3 \mathrm{~nm}$ NPs with 200\% Pd sc (Tables S6-S7), Au, Pd, and Pd-on-Au NP concentration studies (Figure S1-S4), Arrhenius plots of Pd-on-Au NPs (Figures S5-S6), chemical equations for reduction of $\mathrm{PdO}(\mathrm{s})$ by $\mathrm{BH}_{4}{ }^{-}(\mathrm{aq})$ (equations $\mathrm{S} 1-\mathrm{S} 3$ ), and equations used to 
calculate the alloy coordination numbers of $3 \mathrm{~nm}$ Pd-on-Au NPs with $200 \%$ Pd sc (equations S4-

S7).

\subsection{References.}

[1] M.O. Nutt, J.B. Hughes, M.S. Wong, Designing Pd-on-Au Bimetallic Nanoparticle Catalysts for Trichloroethene Hydrodechlorination, Environmental Science \& Technology, 39 (2005) 1346-1353.

[2] M.O. Nutt, K.N. Heck, P. Alvarez, M.S. Wong, Improved Pd-on-Au Bimetallic Nanoparticle Catalysts for Aqueous-Phase Trichloroethene Hydrodechlorination, Appl. Catal., B, 69 (2006) 115-125.

[3] Y.-L. Fang, J.T. Miller, N. Guo, K.N. Heck, P.J.J. Alvarez, M.S. Wong, Structural Analysis of Palladium-decorated Gold Nanoparticles as Colloidal Bimetallic Catalysts, Catal. Today, 160 (2011) 96-102.

[4] L.A. Pretzer, H.J. Song, Y.-L. Fang, Z. Zhao, N. Guo, T. Wu, I. Arslan, J.T. Miller, M.S. Wong, Hydrodechlorination catalysis of Pd-on-Au nanoparticles varies with particle size, Journal of Catalysis, 298 (2013) 206-217.

[5] K.N. Heck, M.O. Nutt, P. Alvarez, M.S. Wong, Deactivation resistance of $\mathrm{Pd} / \mathrm{Au}$ nanoparticle catalysts for water-phase hydrodechlorination, Journal of Catalysis, 267 (2009) 97-104.

[6] Z. Zhao, Y.-L. Fang, P.J.J. Alvarez, M.S. Wong, Degrading perchloroethene at ambient conditions using Pd and Pd-on-Au reduction catalysts, Applied Catalysis B: Environmental, 140-141 (2014) 468-477.

[7] H. Qian, Z. Zhao, J.C. Velazquez, L.A. Pretzer, K.N. Heck, M.S. Wong, Supporting palladium metal on gold nanoparticles improves its catalysis for nitrite reduction, Nanoscale, 6 (2014) 358-364.

[8] K.N. Heck, B.G. Janesko, G.E. Scuseria, N.J. Halas, M.S. Wong, Using Catalytic and SurfaceEnhanced Raman Spectroscopy-Active Gold Nanoshells to Understand the Role of Basicity in Glycerol Oxidation, ACS Catalysis, 3 (2013) 2430-2435.

[9] Z. Zhao, J. Arentz, L.A. Pretzer, P. Limpornpipat, J.M. Clomburg, R. Gonzalez, N.M. Schweitzer, T. Wu, J.T. Miller, M.S. Wong, Volcano-shape glycerol oxidation activity of palladium-decorated gold nanoparticles, Chemical Science, 5 (2014) 3715-3728.

[10] J.C. Velázquez, S. Leekumjorn, Q.X. Nguyen, Y.-L. Fang, K.N. Heck, G.D. Hopkins, M. Reinhard, M.S. Wong, Chloroform hydrodechlorination behavior of alumina-supported Pd and PdAu catalysts, AIChE Journal, 59 (2013) 4474-4482.

[11] Y. Mei, Y. Lu, F. Polzer, M. Ballauff, M. Drechsler, Catalytic activity of palladium nanoparticles encapsulated in spherical polyelectrolyte brushes and core-shell microgels, Chemistry of Materials, 19 (2007) 1062-1069.

[12] S. Wunder, F. Polzer, Y. Lu, Y. Mei, M. Ballauff, Kinetic Analysis of Catalytic Reduction of 4-Nitrophenol by Metallic Nanoparticles Immobilized in Spherical Polyelectrolyte Brushes, Journal of Physical Chemistry C, 114 (2010) 8814-8820.

[13] R. Prucek, L. Kvitek, A. Panacek, L. Vancurova, J. Soukupova, D. Jancik, R. Zboril, Polyacrylate-assisted synthesis of stable copper nanoparticles and copper(I) oxide nanocubes with high catalytic efficiency, Journal of Materials Chemistry, 19 (2009) 84638469. 
[14] K. Kuroda, T. Ishida, M. Haruta, Reduction of 4-nitrophenol to 4-aminophenol over $\mathrm{Au}$ nanoparticles deposited on PMMA, Journal of Molecular Catalysis a-Chemical, 298 (2009) 7-11.

[15] J. Kaiser, L. Leppert, H. Welz, F. Polzer, S. Wunder, N. Wanderka, M. Albrecht, T. Lunkenbein, J. Breu, S. Kuemmel, Y. Lu, M. Ballauff, Catalytic activity of nanoalloys from gold and palladium, Physical Chemistry Chemical Physics, 14 (2012) 6487-6495.

[16] P. Herves, M. Perez-Lorenzo, L.M. Liz-Marzan, J. Dzubiella, Y. Lu, M. Ballauff, Catalysis by metallic nanoparticles in aqueous solution: model reactions, Chemical Society Reviews, 41 (2012) 5577-5587.

[17] T. Aditya, A. Pal, T. Pal, Nitroarene reduction: a trusted model reaction to test nanoparticle catalysts, Chemical Communications, 51 (2015) 9410-9431.

[18] S. Saha, A. Pal, S. Kundu, S. Basu, T. Pal, Photochemical Green Synthesis of CalciumAlginate-Stabilized $\mathrm{Ag}$ and $\mathrm{Au}$ Nanoparticles and Their Catalytic Application to 4Nitrophenol Reduction, Langmuir, 26 (2010) 2885-2893.

[19] J.L. Radomski, PRIMARY AROMATIC-AMINES - THEIR BIOLOGICAL PROPERTIES AND STRUCTURE-ACTIVITY-RELATIONSHIPS, Annual Review of Pharmacology and Toxicology, 19 (1979) 129-157.

[20] H.M. Pinheiro, E. Touraud, O. Thomas, Aromatic amines from azo dye reduction: status review with emphasis on direct UV spectrophotometric detection in textile industry wastewaters, Dyes and Pigments, 61 (2004) 121-139.

[21] A. Kiss, A. Potor, Z. Hell, Heterogeneous catalytic solvent-free synthesis of quinoline derivatives via the Friedlander reaction, Catalysis Letters, 125 (2008) 250-253.

[22] S.C. Mitchell, R.H. Waring, Aminophenols, in: G.D. Considine (Ed.) Van Nostrand's Encyclopedia of Chemistry, Wiley, John \& Sons Inc.2005.

[23] S. Tang, S. Vongehr, G. He, L. Chen, X. Meng, Journal of Colloid and Interface Science, 375 (2012) 125.

[24] D. Zhao, X. Chen, Y. Liu, C. Wu, R. Ma, Y. An, L. Shi, Journal of Colloid and Interface Science, 331 (2009) 109.

[25] J.W. Slot, H.J. Geuze, A New Method of Preparing Gold Probes for Multiple-Labeling Cyto-Chemistry, Eur. J. Cell Biol., 38 (1985) 87-93.

[26] A.Z. Moshfegh, Nanoparticle catalysts, Journal of Physics D-Applied Physics, 42 (2009) 233001.

[27] J.D. Aiken, R.G. Finke, A Review of Modern Transition-metal Nanoclusters: Their Synthesis, Characterization, and Applications in Catalysis, J. Mol. Catal., A, 145 (1999) 1-44.

[28] S. Harish, J. Mathiyarasu, K.L.N. Phani, V. Yegnaraman, Synthesis of Conducting Polymer Supported Pd Nanoparticles in Aqueous Medium and Catalytic Activity Towards 4Nitrophenol Reduction, Catalysis Letters, 128 (2009) 197-202.

[29] S. Wunder, Y. Lu, M. Albrecht, M. Ballauff, Catalytic Activity of Faceted Gold Nanoparticles Studied by a Model Reaction: Evidence for Substrate-Induced Surface Restructuring, Acs Catalysis, 1 (2011) 908-916.

[30] M. Nemanashi, R. Meijboom, Synthesis and characterization of $\mathrm{Cu}, \mathrm{Ag}$ and $\mathrm{Au}$ dendrimer-encapsulated nanoparticles and their application in the reduction of 4nitrophenol to 4-aminophenol, Journal of Colloid and Interface Science, 389 (2013) 260267. 
[31] H.-L. Jiang, T. Akita, T. Ishida, M. Haruta, Q. Xu, Synergistic Catalysis of Au@Ag CoreShell Nanoparticles Stabilized on Metal-Organic Framework, Journal of the American Chemical Society, 133 (2011) 1304-1306.

[32] T. Endo, T. Kuno, T. Yoshimura, K. Esumi, Preparation and catalytic activity of Au-Pd, $\mathrm{Au}-\mathrm{Pt}$, and Pt-Pd binary metal dendrimer nanocomposites, Journal of Nanoscience and Nanotechnology, 5 (2005) 1875-1882.

[33] A.M. Venezia, V. La Parola, V. Nicoli, G. Deganello, Effect of Gold on the HDS Activity of Supported Palladium Catalysts, J. Catal., 212 (2002) 56-62.

[34] F. Gao, Y. Wang, D.W. Goodman, CO Oxidation over AuPd(100) from Ultrahigh Vacuum to Near-Atmospheric Pressures: CO Adsorption-Induced Surface Segregation and Reaction Kinetics, J. Phys. Chem. C, 113 (2009) 14993-15000.

[35] A. Beck, A. Horvath, Z. Schay, G. Stefler, Z. Koppany, I. Sajo, O. Geszti, L. Guczi, Sol Derived Gold-palladium Bimetallic Nanoparticles on $\mathrm{TiO}_{2}$ : Structure and Catalytic Activity in CO Oxidation, Top. Catal., 44 (2007) 115-121.

[36] R.J. Davis, M. Boudart, Structure of Supported PdAu Clusters Determined by X-ray Absorption Spectroscopy, J. Phys. Chem., 98 (1994) 5471-5477.

[37] S.K. Ghosh, M. Mandal, S. Kundu, S. Nath, T. Pal, Bimetallic Pt-Ni nanoparticles can catalyze reduction of aromatic nitro compounds by sodium borohydride in aqueous solution, Applied Catalysis a-General, 268 (2004) 61-66.

[38] A.J. Bard, R. Parsons, J. Jordan, Standard Potentials in Aqueous Solution, Marcel Dekker Inc., New York, 1985, pp. 195.

[39] S. Arora, P. Kapoor, M.L. Singla, Catalytic studies of palladium nanoparticles immobilized on alumina synthesized by a simple physical precipitation method, Reaction Kinetics Mechanisms and Catalysis, 99 (2010) 157-165.

[40] R. Bhandari, M.R. Knecht, Effects of the Material Structure on the Catalytic Activity of Peptide-Templated Pd Nanomaterials, Acs Catalysis, 1 (2011) 89-98.

[41] S. Panigrahi, S. Basu, S. Praharaj, S. Pande, S. Jana, A. Pal, S.K. Ghosh, T. Pal, Synthesis and Size-Selective Catalysis by Supported Gold Nanoparticles: Study on Heterogeneous and Homogeneous Catalytic Process, J. Phys. Chem. C, 111 (2007) 4596-4605.

[42] M. Schrinner, F. Polzer, Y. Mei, Y. Lu, B. Haupt, M. Ballauff, A. Goeldel, M. Drechsler, J. Preussner, U. Glatzel, Mechanism of the formation of amorphous gold nanoparticles within spherical polyelectrolyte brushes, Macromolecular Chemistry and Physics, 208 (2007) 1542-1547.

[43] M.A. Mahmoud, F. Saira, M.A. El-Sayed, Experimental Evidence For The Nanocage Effect In Catalysis With Hollow Nanoparticles, Nano Letters, 10 (2010) 3764-3769.

[44] T. Bligaard, K. Honkala, A. Logadottir, J.K. Norskov, S. Dahl, C.J.H. Jacobsen, On the compensation effect in heterogeneous catalysis, Journal of Physical Chemistry B, 107 (2003) 9325-9331.

[45] E.G. Allison, G.C. Bond, Catalysis Reviews - Science and Engineering, 7 (1972) 233. 